\title{
Design and First Results of SESAM
}

\author{
G. Benner, E. Essers, A. Orchowski and W.-D. Rau \\ LEO Electron Microscopy Group, Carl Zeiss S-M-T AG, D-73446 Oberkochen, Germany
}

We present design and first results of the Sub-Electronvolt-Sub-Ångstrom-Microscope (SESAM) equipped with a Monochromator integrated in the Field Emission Gun (MC-FEG-) and an advanced energy filter, the so- called Mandoline [1].

Fig. 1 shows the CAD model (a) and the principal set-up of the microscope (b). A completely new plinth concept of the microscope has been developed, which is based on supporting the column close to the centre of gravity. The underframe as well as the holding structure has been optimised by FEM analysis in order to achieve the high stability required [2]. The essential modules of this microscope are a 'Field Emission Gun (FEG) with monochromator (MC) and an aberration corrected, high dispersive in-column energy filter. For STEM investigations two High Angular Annular Dark field Detectors (HAADF) as well as 2k SS-CCD camera for acquiring TEM images are available.

Fig. 2 shows the principle set-up of the MC-FEG, which consists of an emitter assembly, a gun lens, the monochromator itself and the multistage accelerator. The electrostatic $\Omega$-type MC according to a proposal of Kahl and Rose provides a dispersion of $12 \mu \mathrm{m} / \mathrm{eV}$ in the symmetry plane and a dispersion free virtual round CO-image [3]. In the MC Off Mode the gun lens delivers a Cross Over (CO) image close to the entrance image plane and the accelerator lens generates a $\mathrm{CO}$ outside the gun. Switching to the MC On mode only a slight readjustment of the gun lens excitation is required for optimum signal transfer and by refocusing the first condenser the spot is kept focussed on the specimen. Due to the optical design, the illumination system inherently preserves spot size while the brightness is only reduced proportional to the amount of beam current cut out by the monochromator [4].

For energy-filtering TEM-applications (EFTEM), the instrument is equipped with an imaging incolumn energy filter. The newly developed Mandoline based on a design by Uhlemann and Rose [1], consists of 1 homogenous and 2 inhomogeneous sector magnet fields and provides full second and third -order correction by means of multipole elements. A superior transmissivity of $\mathrm{T}_{\text {real }}=3300$ nm²/eV@200 kV and $1 \mathrm{eV}$ energy width) is achieved, enabling transfer of highly resolved energy filtered images even at large fields of view. Furthermore the spectrometer provides a dispersion of $6,2 \mu \mathrm{m} / \mathrm{eV} @ 200 \mathrm{kV}$. Combined with the monochromator this makes the instrument to a most powerful tool for ELNES investigations.

\section{References}

[1] S. Uhlemann and H. Rose, (1994) Optik 96, 163

[2] E. Essers, et al., Proc. M\&M 2002, Quebec Canada, Vol. 8, Suppl. 2, 484 CD

[3] F. Kahl and H. Rose, Proc. EUREM Brno/Cz (2000), Vol. III, 1459

[4] M. Haider et al., Proc. $15^{\text {th }}$ Int. Congr. on El. Micr., Durban South Africa (2002)

Corresponding author: Benner@leo.de 


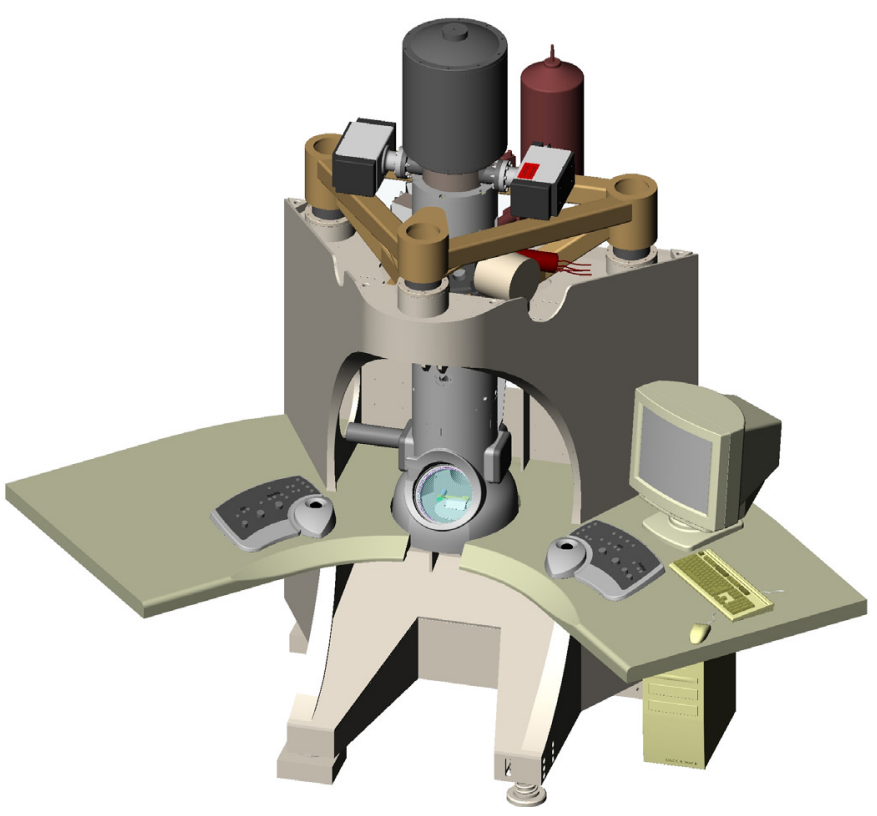

Figure 1: Model of the instrument (a) and the principal optical set-up of the microscope (b)

Figure 2: Principal set-up and asymptotic position of the Cross-Over with Monochromator OFF (a) and Monochromator ON (b)

a)

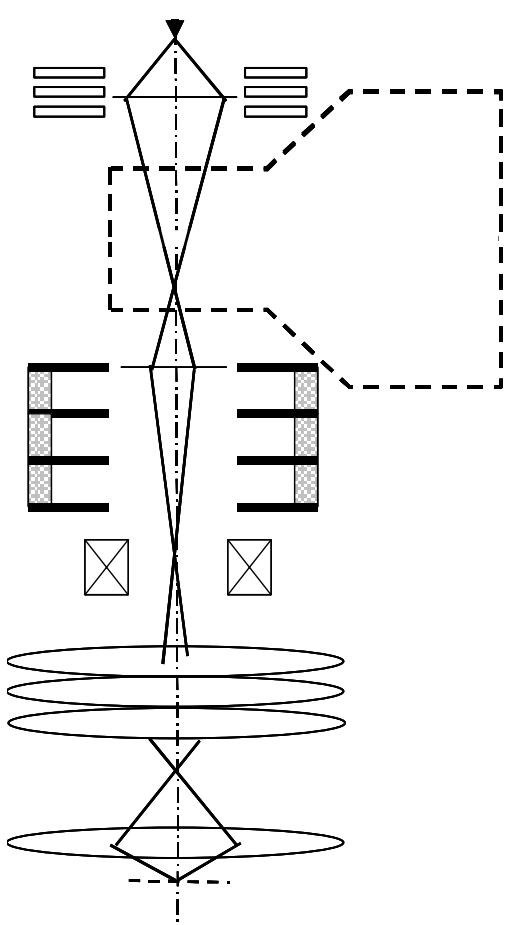

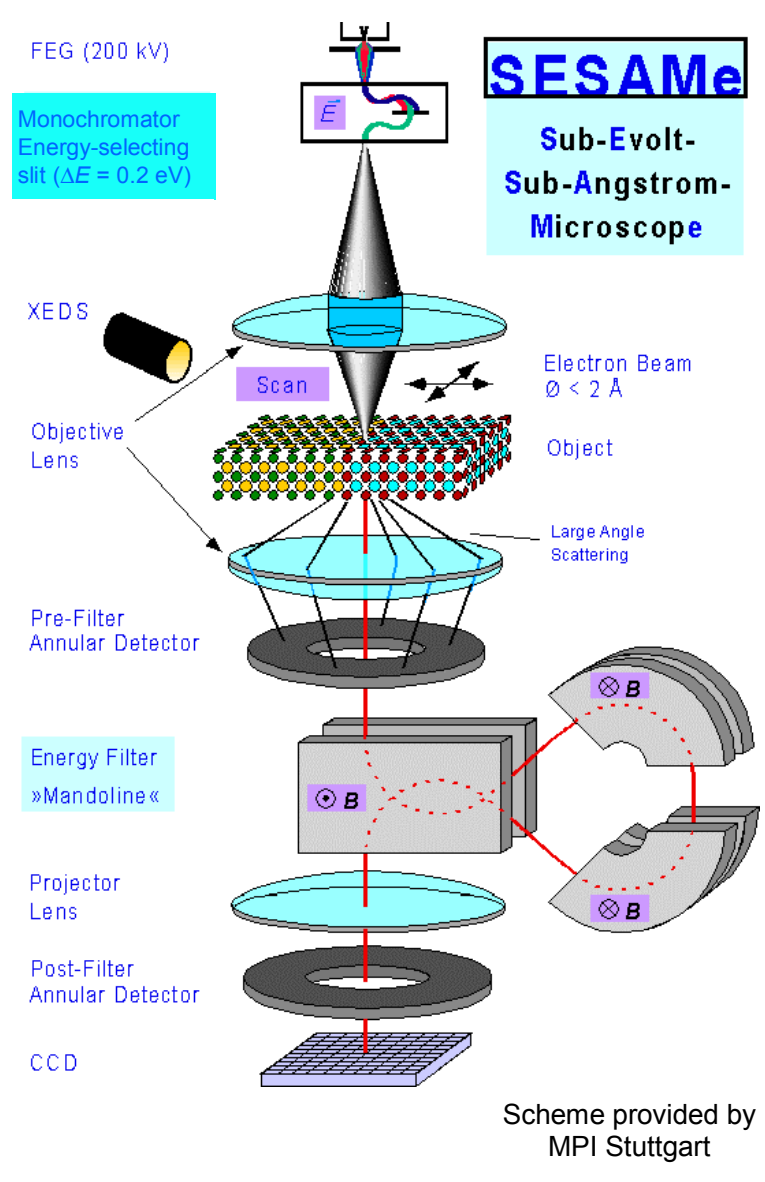

b)

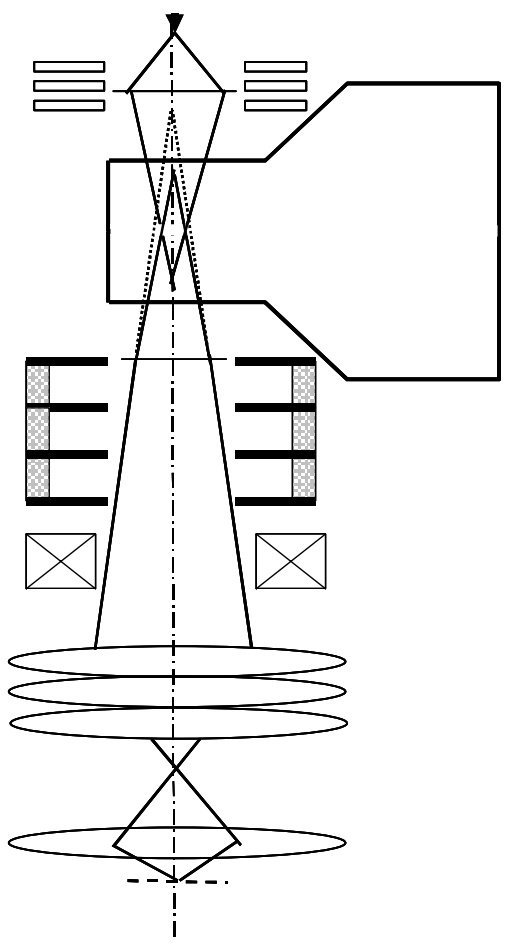

